Chapter 5

\title{
Risk Assessment for Biotic Pests Under Prospective Climate Conditions
}

\author{
Holger Griess, Holger Veit and Ralf Petercord \\ Additional information is available at the end of the chapter \\ http://dx.doi.org/10.5772/56410
}

\section{Introduction}

Mountain ecosystems are among the most species-rich sites on the planet and are particularly sensitive to climatic changes. Although the Alps are certainly the best-studied mountain region in the world, long-term studies of species distribution are still hard to find. In particular, there are no comparative studies covering the Alpine region as a whole. Climate change will affect the mountain forests, on the one hand through direct impacts such as longer drought periods, increased storm damage risk, and on the other hand, through indirect effects such as the development of forest pests. Temperatures in the European Alps have increased twice as much as the global average temperature since the late nineteenth century and are predicted to rise by an average of $0.3-0.5^{\circ} \mathrm{C}$ per decade in the next century. The effects of a rising temperature will influence the occurrence and the impact of biotic risks. A suite of biotic and abiotic factors, ranging from climatic variables and edaphic conditions, to competition, predation and resource distribution, have been invoked to explain the limits in species distributions. Still, our understanding of the spatial dynamics at range limits and their underlying factors is far from complete. Our ability to predict the ecological and evolutionary responses of organisms to climate change requires understanding of the mechanistic links between climate and geographical range limits. The consequences of climate change may involve expansion, contraction, or shifts in species range, and some species may migrate to new areas and exploit previously unutilized resources. The latter situation can only occur if conditions in the new part of the range have been or have become suitable; for example, global warming can allow temperature-limited species to colonize latitudes or altitudes beyond their original distribution. Within this Project we concentrated our efforts on the following two questions:

Will the effects of climate change promote a migration of forest pests from south to more northerly alpine regions? 
Does the changing climate increase the habitat suitability for quarantine organisms?

\section{Forest pest migration - The pine processionary moth}

An increasing number of taxa are undergoing significant range shifts in response to humanassisted dispersal and changes in environmental factors, notably. Often these range shifts are into novel environmental space, from both biotic and abiotic perspectives.

Over the last century, climatic isotherms over Europe have been displaced by an average of $120 \mathrm{~km}$ northwards. A latitudinal and altitudinal expansion has been documented in several important forest pest species in the northern hemisphere, for instance Thaumetopoea pityocampa (pine processionary moth) in central Europe. The pine processionary moth is a monovoltine conifer defoliator, widespread in the Mediterranean region. Adults emerge in the summer, mate and lay eggs within 2-4 days. The gregarious larvae hatch after ca. 1 month and their development progresses until late winter, with the larvae sheltering inside self-spawn silk nests. Pupation takes place in the soil, where the pupae undergo an obligatory diapause. T. pityocampa exhibits a variable phenology across its range of distribution, with late adult emergence (August-September) and early pupation (January-February) occurring in the warmest regions, whereas early adult emergence (June- July) coupled with late pupation (March-April), is observed in populations colonizing the northernmost, or high-altitude areas.

In the last three decades, substantial expansion of the range of this economically important pine defoliator has taken place both latitudinal and altitudinal. In the Paris Basin the range boundary shifted by $87 \mathrm{~km}$ northwards between 1972 and 2004; in the Italian alpine region, an altitudinal shift of 110 - $230 \mathrm{~m}$ upwards occurred between 1975 and 2004.

\section{Increase habitat suitability - The emerald ash borer}

The emerald ash borer, Agrilus planipennis, is one of the most feared beetles. It has the highest quarantine status. National Plant Protection Organizations (NPPOs) around the world continuously inspect their indigenous Fraxinus trees and imported wood products on the presence of this beetle because its presence usually results in the destruction of Fraxinus trees.

Agrilus planipennis belongs to the family of the Buprestidae, also called metallic wood-boring beetles. Buprestids are relatively small, elongated beetles. The emerald ash borer measures approximately 7.5-15 mm. Its larvae measures about 15-30 mm. Females lay eggs on the bark of the host trees in May-June. One female lays about 65-90 eggs during her lifetime. Eggs hatch and the larvae bore to the phloem area just behind the bark, where they create serpentine tunnels. The development through four larval stages takes about 1-2 years depending on the vigor of the host trees in temperate zones. Fourth instar larvae excavate chambers either in the bark or slightly in the sapwood, where they become prepupa in September-October. Most of the population overwinters in this stage, although some individuals overwinter as earlier- 
instar larvae. In these chambers, they develop into pupae during spring, and emerge as adults in May/June. The adults live approximately 2-3 weeks, during which they disperse no more than several kilometers. Host plants of A. planipennis are almost exclusively Fraxinus trees: in northern America they include F. americana, F. chinensis, F. japonica, F. lanuginose, and F. nigra, but the emerald ash borer was also found in Ulmus davidiana var. japonica, Ulmus propinqua, Juglans mandshurica var. sieboldiana, and Pterocarya rhoifolia. In Europe host trees are F. excelsior, F. pennsylvanica, and possibly F. angustifolia.

The native distribution of A. planipennis includes Mongolia, central, eastern, and north-eastern China, Taiwan, both Koreas and Japan. In July 2002 the species was found in Northern America: specimens were identified in south-eastern Michigan, USA. By March 2009, emerald ash borer infestations had been found in a total of ten states and two Canadian provinces. Estimates indicate that more than 53 million native ash trees had been killed by A. planipennis in those states by 2007.

It is believed that the species entered the USA at Detroit, in freight from cargo ships. In Europe, the species was found in Moscow, Russia in 2005 and seems to be spreading. In city squares or along railway tracks many Fraxinus trees are declining or dying. In some places, $70-80 \%$ of the Fraxinus trees have lost most of their foliage. The pathway of this introduction is not known. The species has not been found in other areas of Europe.

The species damages infested trees because the larvae bore serpentine tunnels in the cambial layer and the inner bark (phloem), disrupting, or even completely cutting off the nutrient flow from the leaves to the roots. During the early stage of an infestation, when A. planipennis population is low, the initial damage is low. However, after 2 to 3 years of continuous infestation, the population builds up, and eventually the tree's nutrient and water transport system is disrupted, culminating in wilting and eventual tree mortality. A. planipennis will kill apparently healthy trees during high beetle population levels which are probably triggered by a few years of hot and dry climatic conditions. A. planipennis can cause severe damage to ash stands over eight years of age that are not crown-closed, with good sun light penetration, and that are comprised of trees with bark fractures. After one to two years of infestation, the bark often falls off in pieces from damaged trees thereby exposing the tunnel-ridden sapwood. In the most severe cases, entire stands may be destroyed. In its natural area, A. planipennis population dynamics are balanced due to natural enemies: parasitoids, predators, and entomopathogenic fungi. Apparently, the absence of most of these natural enemies in the 'new' areas has an enormous impact on the population dynamics, allowing build-up of A. planipennis populations to such high levels as to become a true pest.

Other symptoms that may indicate an A. planipennis infestation and may be seen from the outside, include crown dieback/chlorosis, epicormic shoots, increased woodpecker and squirrel feeding, bark deformities, foliage feeding, and exit holes. The exit holes are D-shaped of 3.5-4.1 mm in diameter. A clear sign of an Agrilus sp. infestation that cannot be seen from the outside is the presence of typically serpentine shaped larval galleries, mostly filled with shavings. They can easily be found by peeling away the bark. Once the signs and symptoms become apparent, the trees are often severely infested. The infestation may have spread to surrounding areas where signs are not yet apparent. Based on the infestation symptoms, 
examination techniques applied by inspectors should include visual inspection, crown survey, and bark peeling. The most effective way to control and eradicate an outbreak of A. planipennis is by felling and chipping or burning infested trees. This should be done in the infested and as a preventive measure in the surrounding area.

The fact that ashes are very common in the alpine region and that the species has already entered Eastern Europe poses a serious phytosanitary risk.

\section{Species distribution model}

Global climate change is ever more evident. Consequently, geographic areas corresponding to biomes, ecosystems and species' ecological niches are changing, which is likely to affect the distribution of many species. Species distribution modeling can be used to provide a rapid evaluation of the potential impact of climate change on the distribution of ecosystems and the species that inhabit them. The process consists of detecting changes in species distribution by comparing the potential distribution areas in the current climate with the potential distribution areas, based on a species' current climate preferences, under future climatic conditions. Future potential distribution areas of occurrence are identified using climate layers based on the projections of General Circulation Models (GCM). Climate research institutions from various countries generate these which predict future climatic conditions under different emission scenarios developed by the Intergovernmental Panel on Climate Change (IPCC).

The environmental data for the global climatic conditions was obtained from the WorldClim database, which provides 19 layers of bioclimatic variables (Table 1) compiled from monthly data. The data for the present day conditions was collected from 1950 to 2000 with a spatial resolution of $30 \operatorname{arcsec}\left(1 \mathrm{~km}^{2}\right)$. For the niche models under future conditions WorldClim data was obtained for the years 2020, 2050 and 2080. Prior to use, the WorldClim grids were fitted in Arc-GIS to the extent of the natural and invaded range. This allows the projection of the models into the area of interest. In addition to the climatic data we added altitude data for the whole range.

A commonly employed method for risk assessment with regard to range shifts and range extension of invasive species is climate-based ecological niche modeling. It has been used to predict potential ranges for recent forest invaders in North America e.g. the Asian longhorn beetle However, despite its possible ecological and economic impact there is no climate-based model of the potential large-scale distribution of the emerald ash borer in the alpine region.

To determine the potential geographic distribution for T. pityocampa and A. planipennis in the alpine region we used ecological niche modeling procedures. Ecological niche modeling involves generating a set of predictive rules that describe relationships between a species georeferenced occurrence and a suite of spatially explicit environmental variables, most commonly bioclimatic factors such as temperature and precipitation.

The basis for spatial analysis of species distribution is occurrence data. Occurrence data is a snapshot of species in time and space. To analyze occurrence data, we used the data provided 


\begin{tabular}{|c|c|}
\hline Bioclimatic Variable & Description \\
\hline $\mathrm{BIO} 1$ & Annual Mean Temperature \\
\hline $\mathrm{BIO} 2$ & Mean Diurnal Range (Mean of monthly (max temp - min temp)) \\
\hline $\mathrm{BIO} 3$ & Isothermality (BIO2/BIO7) (* 100) \\
\hline $\mathrm{BIO} 4$ & Temperature Seasonality (standard deviation *100) \\
\hline $\mathrm{BIO5}$ & Max Temperature of Warmest Month \\
\hline $\mathrm{BIO6}$ & Min Temperature of Coldest Month \\
\hline $\mathrm{BIO7}$ & Temperature Annual Range (BIO5-BIO6) \\
\hline $\mathrm{BIO8}$ & Mean Temperature of Wettest Quarter \\
\hline $\mathrm{BIO9}$ & Mean Temperature of Driest Quarter \\
\hline $\mathrm{BIO} 10$ & Mean Temperature of Warmest Quarter \\
\hline $\mathrm{BIO} 11$ & Mean Temperature of Coldest Quarter \\
\hline $\mathrm{BIO} 12$ & Annual Precipitation \\
\hline $\mathrm{BlO} 13$ & Precipitation of Wettest Month \\
\hline $\mathrm{BIO} 14$ & Precipitation of Driest Month \\
\hline $\mathrm{BIO15}$ & Precipitation Seasonality (Coefficient of Variation) \\
\hline $\mathrm{BIO} 16$ & Precipitation of Wettest Quarter \\
\hline $\mathrm{BIO} 17$ & Precipitation of Driest Quarter \\
\hline $\mathrm{BIO18}$ & Precipitation of Warmest Quarter \\
\hline $\mathrm{BIO19}$ & Precipitation of Coldest Quarter \\
\hline
\end{tabular}

Table 1. Structure of bioclimatic data used for modeling

by our project partners and publicly available data provided through international platforms. Much of the available data are historical observations which may not reflect the current presence of taxa due to recent ecological processes or human interventions, such as forest conversion to agriculture and other changes in land use. Nonetheless, such data is still useful to gain insights into the ecological processes behind the geographical distribution of species distribution. Observation data must be organized following the format specified by the applied niche modeling software. The analyses can be based on presence or absence data or a combination of both. Basic data consists of a taxonomic identification of an observed individual and the location of the collection or observation site. Presence points may be associated with additional data describing the date of collection, source of coordinates. Information about the collection date provides a time dimension to the analyses and can be used to analyze trends in species distribution.

The opposite of presence points is absence data. While absence data can also be relevant in spatial analyses, e.g. monitoring trends in species distribution, it is often challenging to understand concrete reasons for the absence of taxa in a geographic unit, complicating the use 
of this data in ecological analyses. Therefore, the ecological niche modeling for this project is based on presence points only.

Often, the available set of presence data does not cover the entire range of a species' natural distribution. Building a reliable ecological niche model can be problematic, since only limited information is available on the native range and out of range occurrence of the species. Sources claim that e.g. the emerald ash borer may be native to northeastern China, however documented observations and museum specimens are scarce. It was possible to identify 496 georeferenced occurrence records for A. planipennis and 169 for T. pityocampa from various sources. Species distribution modeling programs such as Maxent (PHILLIPS et al. 2004, 2006) enable us to approximate the full distribution range. These programs are practical tools to identify those areas where a species is likely to occur. The results of the species distribution modeling analysis can be used for different combined spatial analyses, e.g. evaluating the impact of climate change on the future distribution of species. Species distribution modeling programs identify sites with similar environments to those where a species has already been observed as potential occurrence areas. The data required to identify these potential distribution areas include species presence points as well as the raster data of environmental variables covering the study area. First, a niche is defined based on the environmental values that correspond to the presence points used in the analysis. Then, the similarities between the environmental values at a specific cell in the data raster and those of the niche of the modeled species are calculated for each raster cell in the study area. With this information, the model calculates the probability of a species occurrence in each raster cell. Even though the next analysis is based on climate data, Maxent also allow us to include other types of variables in the model such as altitude.

Maxent calculates the species' realized niche and probability of occurrence using an algorithm for maximum entropy. Since Maxent has fared well in evaluations, in comparison to other niche modeling programs, it is the program of choice in this project.

Maxent is a general-purpose method for making predictions or inferences from incomplete information. Its origins lie in statistical mechanics, and it remains an active area of research with an Annual Conference, Maximum Entropy and Bayesian Methods, that explores applications in diverse areas. The idea of Maxent is to estimate a target probability distribution by finding the probability distribution of maximum entropy (i.e., that is most spread out, or closest to uniform), subject to a set of constraints that represent our incomplete information about the target distribution. The information available about the target distribution often presents itself as a set of real-valued variables, called "features", and the constraints are that the expected value of each feature should match its empirical average (average value for a set of sample points taken from the target distribution). When Maxent is applied to presence-only species distribution modeling, the pixels of the study area make up the space on which the Maxent probability distribution is defined, pixels with known species occurrence records constitute the sample points, and the features are climatic variables, elevation and functions thereof. Maxent offers many advantages. It requires only presence data, together with environmental information for the whole study area. It can utilize both continuous and categorical data, and can incorporate interactions between different variables. Efficient deterministic algorithms 
have been developed that are guaranteed to converge to the optimal, maximum entropy, probability distribution. The Maxent probability distribution has a concise mathematical definition, and is therefore amenable to analysis. For example, as with generalized linear and generalized additive models (GLM and GAM), in the absence of interactions between variables, additivity of the model makes it possible to interpret how each environmental variable relates to suitability. Over-fitting can be avoided by using regularization. The output is continuous, allowing fine distinctions to be made between the modeled suitability of different areas. If binary predictions are desired, this allows great flexibility in the choice of threshold. Maxent is a generative approach, rather than discriminative, which can be an inherent advantage when the amount of training data is limited.

It is important to realize that when a geographical area shows environmental conditions favorable for a species, this does not necessarily mean that the species actually occurs in this area.

\section{Results}

The range of the pine processionary moth under present day climatic conditions in the Alpine region is illustrated in Figure 1. The habitat suitability is very low across the Alpine arc. Only two departments in France show a higher suitability. In the department of Rhône-Alps areas with low habitat suitability are evident the westernmost parts. A low to medium habitat suitability can be observed in the southern part of the department of Provence-Alpes-Côte D 'Azur.

The habitat suitability under possible climate conditions in the future is pictured in Figure 2. The overall habitat suitability is still very low across the Alpine arc. The area with a low suitability has increased in the departments of Rhône-Alps and Provence-Alpes-Côte D 'Azur. Yet for the latter department the suitability decreased from medium-low to low.

Under present day climate conditions our models show very low habitat suitability across the Alpine region for the emerald ash borer. The exception is a small area in the east of the department Rhône-Alpes where low habitat suitability can be observed (Figure 3).

The results of our model for the habitat suitability of the emerald ash borer under conditions influenced by climate change are illustrated in Figure 4. In comparison to the present day condition we can observe a significant increase of area with low and medium habitat suitability. This increase in area is concentrated in the German and Austrian part of the Alps.

\section{Discussion}

With the results of our models we can now return to the beginning and answer the questions.

- Will the effects of climate change promote a migration of forest pests from south to more northerly alpine regions? 


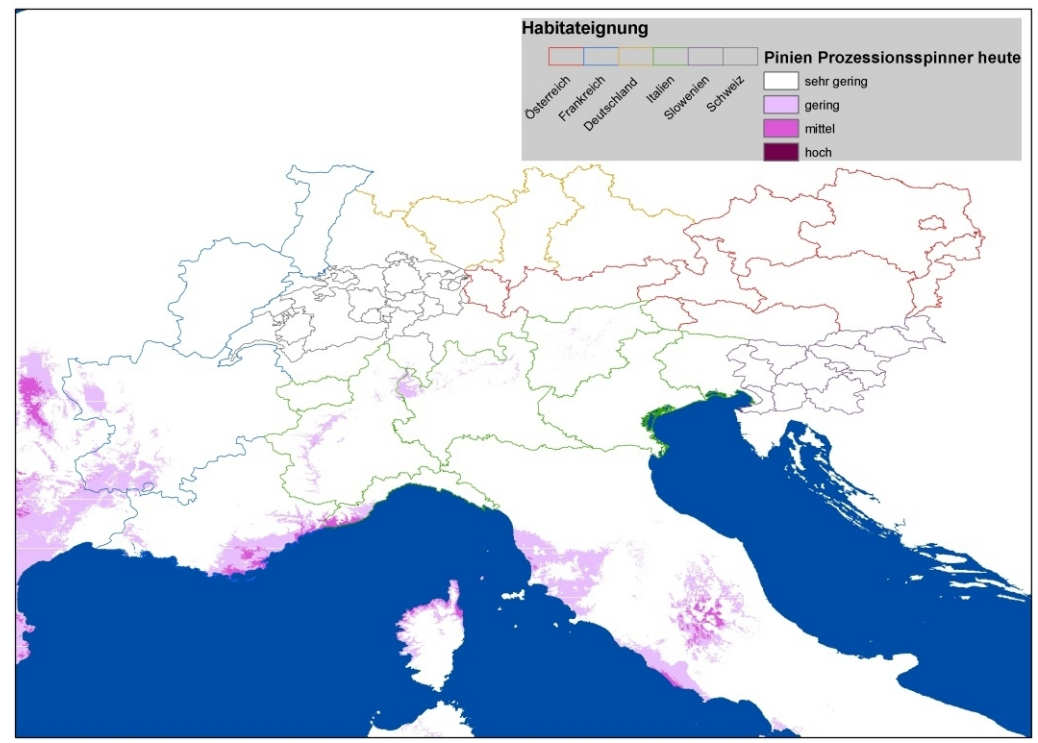

Figure 1. Potential habitat suitability Pine Processionary Moth in the Alpine region under present climatic conditions

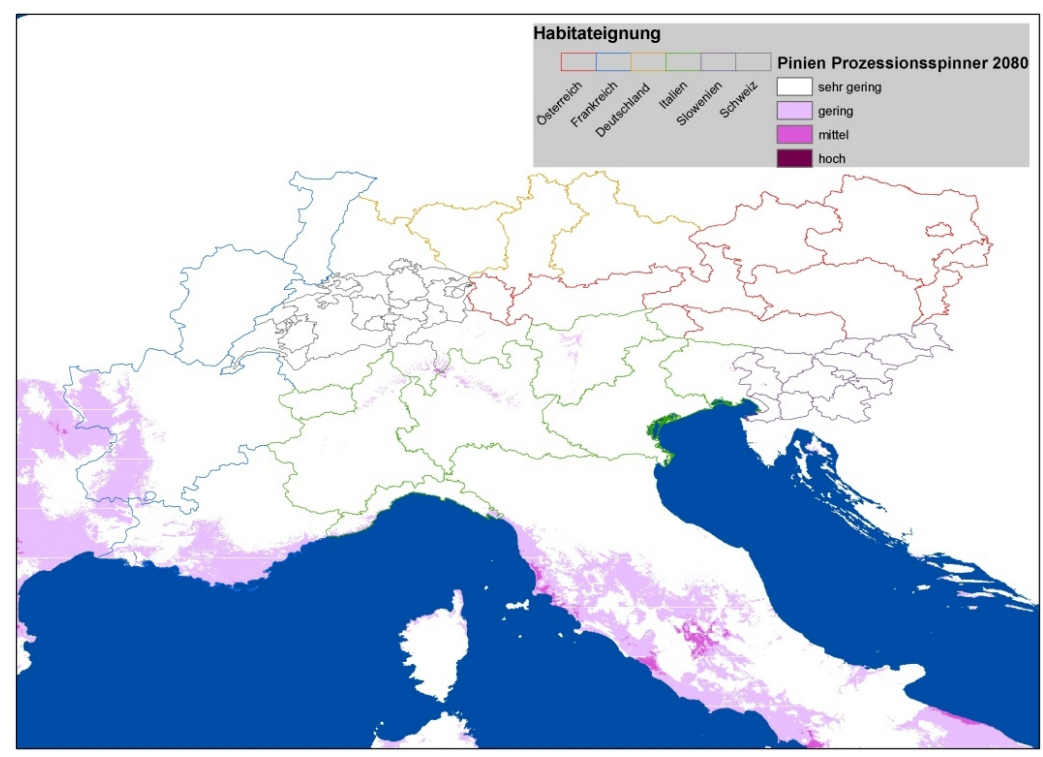

Figure 2. Potential habitat suitability Pine Processionary Moth in the Alpine region under possible climatic conditions in 2080 


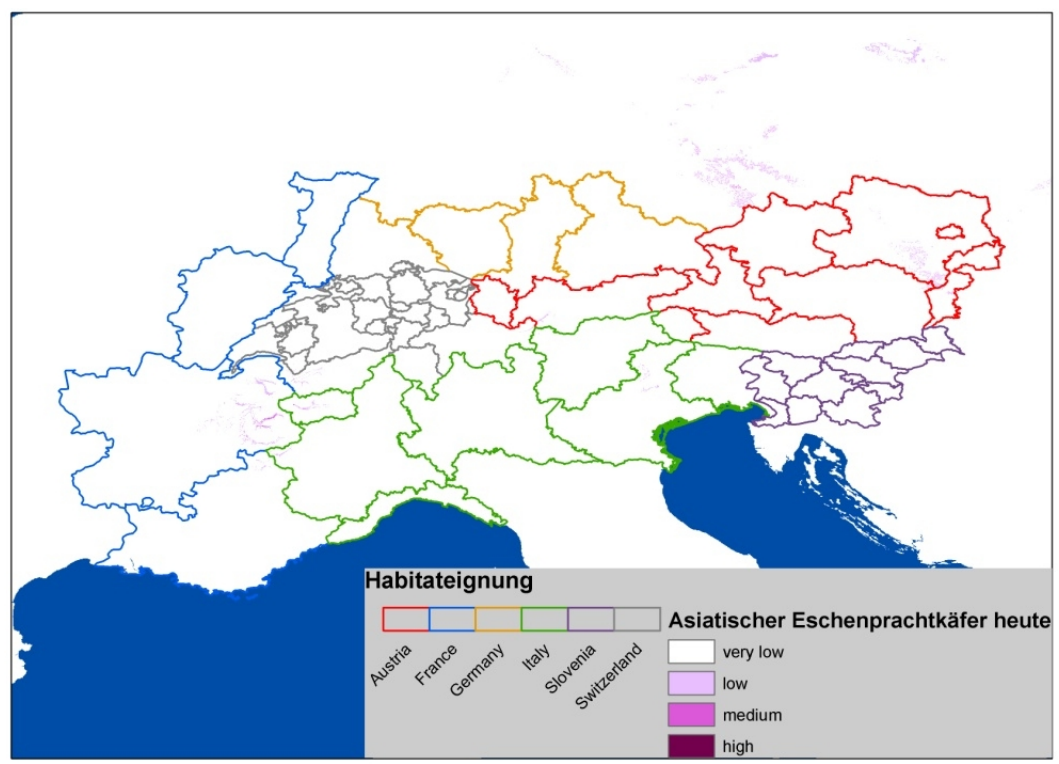

Figure 3. Potential habitat suitability Emerald Ash Borer in the Alpine region under present climatic conditions

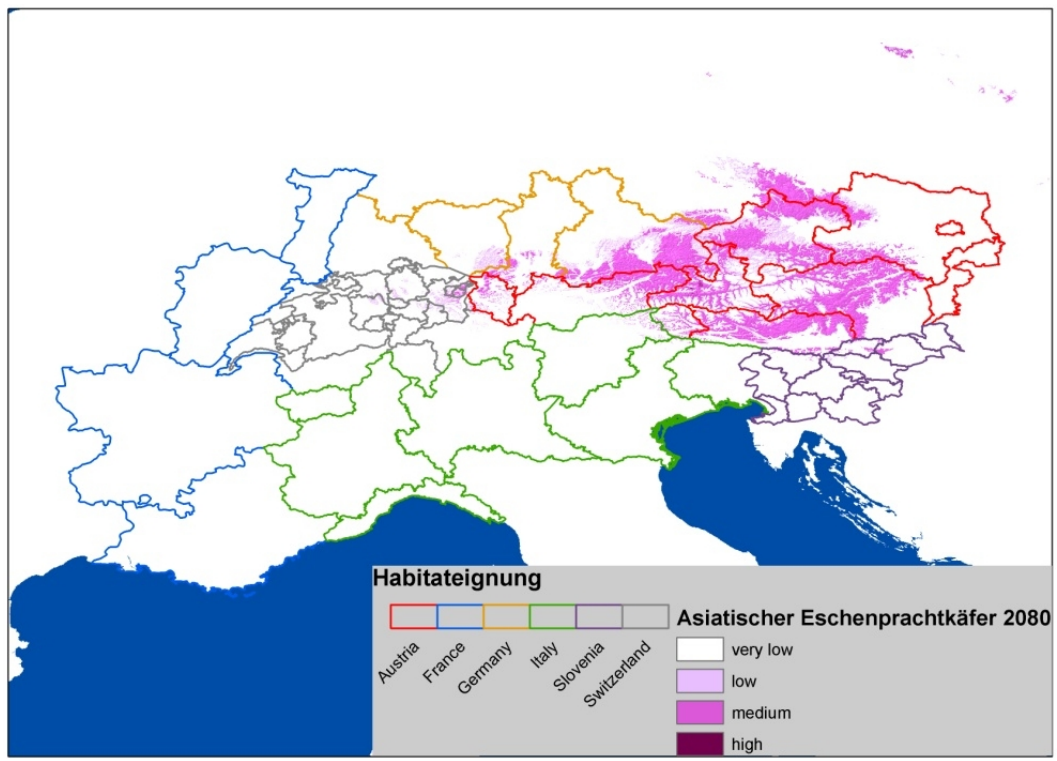

Figure 4. Potential habitat suitability Emerald Ash Borer in the Alpine region under potential climatic conditions 2080 
- Does the changing climate increase the habitat suitability for quarantine organisms?

In case of the pine processionary moth as an example for a forest pest with potential to migrate from south to north, our models show that a migration is possible under changing climate conditions in the future. Yet the range enlargement is not as big as other studies, which base their models solely on a rise in temperature, imply. Our models show that the main factors for a possible range enlargement for $T$. pityocampa are the temperature seasonality, the annual range of temperature and the mean temperature in the coldest quarter. To better understand why these factors are important in the habitat suitability of T. pityocampa we need to cross validate them biology of T. pityocampa. The pine processionary moth has been worked on in many scientific papers, the knowledge of the biology of it is still limited. We need to increase that knowledge in order to make the results for the species distribution modeling for the pine processionary moth more precise.

Even if we can't see a dramatic change according to our model, a higher impact of thaumetopoea processionea is still possible. For sites with less favorable climate conditions, like southern slopes in the alpine region, an infestation with T. pityocampa might have severe consequences. These sites are usually so dry that water is a limiting factor for tree growth. Pines are the only trees which are able to compete under these conditions. The pines are under constant water stress which makes the susceptible to pests. An infestation with T. pitycampa might lead to a destabilization of the whole stand and may cause the loss of forest functions in that area.

The emerald ash borer is a feared quarantine pest organism in Europe. An occurrence and spread can have serious ecological and economic consequences. Our models show that for the northern part of the Alps a changing climate will lead to a more suitable habitat. A more suitable habitat for the emerald ash borer increases the risk of an outbreak after an initial infection with this quarantine organism.

Due to the inner European trade routes and tourism the alpine region has a high road density. The number of vehicles which are traveling across the whole alpine space is increasing every year. Traffic is a possible pathway for an infection with the emerald ash borer. An increase in traffic in the future combined with higher habitat suitability due to climate change will lead to high possibility of the occurrence and outbreak of the emerald ash borer in the alpine region.

In order to decrease the chance of a possible outbreak a better monitoring system in the areas with a higher suitability is needed. Within the Manfred-Project an internet based monitoring platform has been developed. This platform allows a quick exchange of information between the forest protection specialists in the alpine region. This helps to spread the information about a detected infection in the earliest possible stage and minimizes the time to take counteractions against the thread. 


\section{Author details}

Holger Griess ${ }^{1}$, Holger Veit ${ }^{2}$ and Ralf Petercord ${ }^{1}$

1 Landesanstalt für Wald und Forstwirtschaft Bayern (LWF), Freising, Germany

2 Forstliche Versuchs- und Forschungsanstalt Baden-Württemberg (FVA), Department of Biometrics, Freiburg im Breisgau, Germany

\section{References}

[1] Phillips, S. J., Dudik, M. \& Schapire, R.E. 2004. A maximum entropy approach to species distribution modeling. Pages 655-662 in Proceedings of the 21st International Conference on Machine Learning. ACM Press, New York

[2] Phillips, S. J., R. P. Anderson, and R. E. Schapire. 2006. Maximum entropy modeling of species geographic distributions. Ecological Modelling, 190:231-259. 
